\title{
Segmental torsion assessment is a reliable method for in-depth analysis of femoral alignment in Computer Tomography
}

\author{
Peter Wilhelm Ferlic ${ }^{1}$ (D) Armin Runer ${ }^{1} \cdot$ Christopher Seeber $^{1} \cdot$ Maria Thöni $^{2}$ • \\ Gerd Seitlinger $^{3} \cdot$ Michael Christian Liebensteiner $^{1}$
}

Received: 8 May 2017 / Accepted: 31 July 2017 / Published online: 14 August 2017

(C) The Author(s) 2017. This article is an open access publication

\begin{abstract}
Purpose De-rotational osteotomies are indicated in patients with pathologic femoral torsion. However, there is disagreement whether an osteotomy should be performed proximally or distally. Conventionally only the total torsion is measured, which does not allow differentiation between a torsional deformity located in the proximal or distal metaphysis or the diaphysis. The aim of this study is to validate a new multilevel measurement protocol for evaluation of the magnitude of torsion of the respective femoral segments in CT.

Patients and methods The torsional profile of 30 femora was evaluated in CT scans. For separate measurements of the torsion of the metaphysis and the diaphysis, four axes where determined: one through the femoral neck, a second determined by the midpoint of the femoral shaft and the lesser trochanter, a third determined by a tangent dorsal to the popliteal surface, and a fourth axis posterior to the condyles. The total femoral torsion was measured between the first and the fourth axis, proximal torsion between the first and the second, mid torsion between the second and the third, and distal
\end{abstract}

Investigation performed at the Medical University of Innsbruck, Innsbruck, Austria.

Peter Wilhelm Ferlic

peter.ferlic@gmail.com

Armin Runer

armin.runer@rolmail.net

Christopher Seeber

seeber.ch@gmx.at

Maria Thöni

maria.thoeni@tirol-kliniken.at

Gerd Seitlinger

g.seitlinger@gmx.de torsion between the third and the fourth axis. Four investigators performed all measurements independently and intraclass correlation coefficients (ICC) were calculated to evaluate intra- and inter-rater reliability.

Results Average total femoral torsion was $22.6 \pm 8.7^{\circ}$, proximal torsion $47.7 \pm 10.6^{\circ}$, mid torsion $-33.4 \pm 9.9^{\circ}$, and distal torsion $8.3 \pm 3.2^{\circ}$. Intra-rater ICC ranged between 0.504 and 0.957 and inter-rater ICC between 0.643 and 0.992 . The majority of the ICC were graded as "almost perfect" and some as "substantial" agreement.

Conclusion Evaluation of the segmental torsion of the femur allows in-depth analysis of femoral alignment. High reliability was shown for this measuring method in computed tomography, which can be deployed when studying interdependencies between joint pathologies and torsional deformities or when planning the site for an osteotomy.

Level of evidence Level III.

Keywords Femoral antetorsion - Femoral torsion Reliability $\cdot$ Patellofemoral · Osteotomy

Michael Christian Liebensteiner michael.liebensteiner@i-med.ac.at

1 Department of Orthopaedic Surgery, Medical University of Innsbruck, Anichstrasse 35, 6020 Innsbruck, Austria

2 Department of Radiology, Medical University of Innsbruck, Innsbruck, Austria

3 General Hospital Oberndorf, Teaching Hospital of Paracelsus Medical University Salzburg, Oberndorf, Austria 


\section{Introduction}

Torsional deformities of the femur have an impact on the biomechanics and forces acting in the hip and knee and play a significant role in the development of disorders of these joints [1-3]. Deformities of the femur have been treated with de-rotational osteotomies for many years $[4,5]$. While the treatment of deformities in frontal or sagittal plane is based on well-established rules drawing on the concept of the centre of rotation of angulation (CORA) [6, 7], there are no specific treatment rules for femoral torsional deformities. Hence, disagreement still prevails on whether an osteotomy should be performed proximally or distally in patients with pathologic femoral torsion $[8,9]$. Knowing the segment of the femur in which a torsional deformity is actually localized (i.e., concept of CORA) could help us in this discussion and eventually improve treatment guidelines.

Consequently, the aim of this study was to adapt and validate a recently published measurement protocol for the determination of femoral torsion, which should make it possible to detect the part of the femur in which the torsional deformity is localized, instead of measuring femoral torsion solely between the proximal and distal ends of the femur $[3,10]$. It was hypothesized that this multi-level measurement protocol is a reliable tool for evaluation of the magnitude of torsion of the respective femoral segments and for differentiation of their contribution to overall femoral torsion.

\section{Methods}

After obtaining the approval of the ethics committee of the medical university a retrospective analysis of all lower limb CT scans available in our hospital's digital patient archiving system for the period between 2007 and 2013 was performed. This covered a total of 166 multislice CTs of lower limbs performed in a helical scan technique without contrast media. The radiologic examinations with a $2.5-\mathrm{mm}$ slice thickness were conducted at the level of the hips, knees, and ankles including a CT scanogram of the lower limbs. The patients were scanned in the supine position with the knee fully extended; 51 CT scans of patients with previous operations or fractures in the region of interest were excluded from this study. Of the remaining 115 cases 15 women and 15 men, in total 30 cases with a mean age of $24.8 \pm 8.4(15.1-45.3)$ years, were randomly selected. These 30 cases had deformities, mainly post-traumatic, on one leg and the measurements were done on the contralateral healthy side.

\section{Parameters of femoral torsion}

To allow differentiation of the torsion of the proximal and distal metaphysis as well as the diaphysis, four axes where determined. The first axis was defined by the centre of the femoral head and neck on superimposed transverse slices as described by Murphy et al. [11]: the centre head on one transverse slice was connected to the centre of an ellipse around the base of the femoral neck on another transverse slice. The centre of the lesser trochanter at its greatest prominence and the center of the femoral shaft were used as starting and end points for a line defining the second axis. The third axis was defined by a tangent to the dorsal part of the distal femur in the transverse slice just above the point of attachment of the gastrocnemius heads and the fourth axis by a tangent to the posterior condyles (Fig. 1) [3, 10].

The total femoral torsion was measured between the first and the fourth axis of the femur, proximal torsion between the first and the second axis, mid torsion between the second and the third axis, and distal torsion between the third and the fourth axis. A medial twist of the femur was defined as a positive, a lateral twist as a negative value.

\section{Statistics}

To evaluate the intra- and inter-rater reliability of total and segmental torsion, the 30 cases included were independently evaluated by four investigators and all measurements were repeated by each investigator after one month. For descriptive statistics the mean torsion values of each case were analyzed.
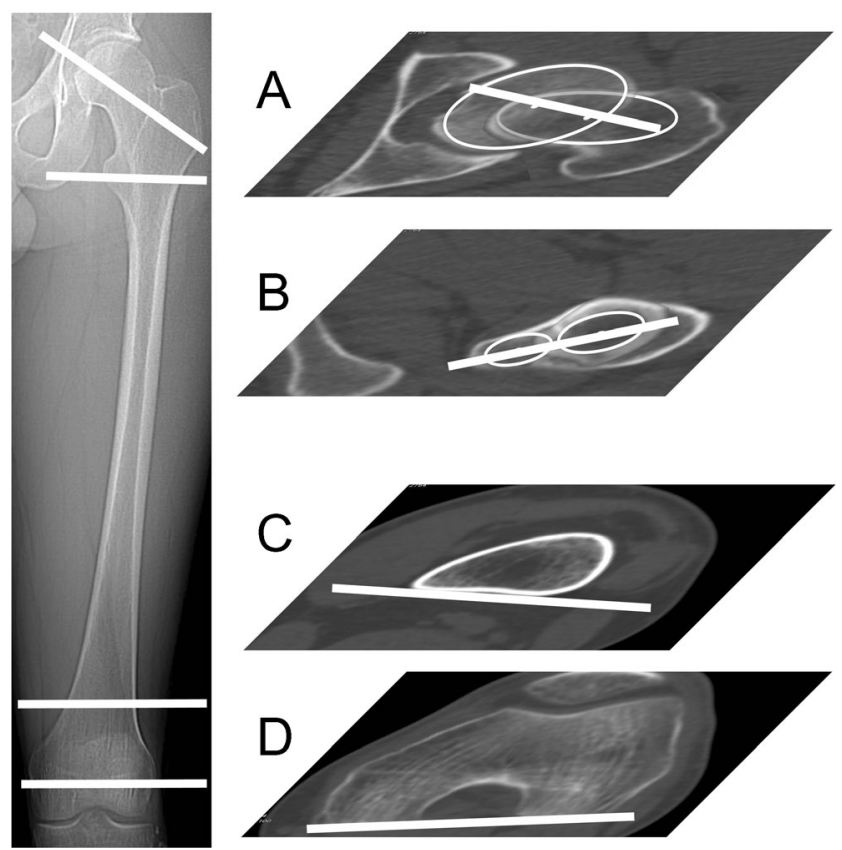

Fig. 1 Determination of the four axes for segmental measurement of femoral torsion. The first axis (a) is determined by the centre of the femoral head and neck, the second axis (b) by the midpoint of the femoral shaft and the lesser trochanter, the third axis (c) by a tangent dorsal to the popliteal surface just proximal to the gastrocnemius muscle, and the fourth axis (d) by a tangent posterior to the femoral condyles 
Results are given as: mean \pm standard deviation (range). A Shapiro-Wilk test was done to prove normal distribution and gender differences were assessed using the t-test. Inter-rater reliability (IRR) was assessed to demonstrate consistency of the measurements [12] using the intraclass correlation coefficient (ICC), which is a widely used reliability index in testretest, intra-rater, and inter-rater reliability analyses [13]. The two way mixed ICC were calculated to evaluate the intra(single measure) and inter-rater (single and average measures) reliability. Intra- and inter-rater agreement was graded according to Landis and Koch [14], who characterized values $<0$ as indicating no agreement and $0-0.20$ as slight, $0.21-0.40$ as fair, $0.41-0.60$ as moderate, $0.61-0.80$ as substantial, and $0.81-1$ as almost perfect agreement.

\section{Results}

Average total femoral torsion was $22.6 \pm 8.7^{\circ}(10.2-42.8)$, proximal torsion $47.7 \pm 10.6^{\circ}(27.6-64.5)$, mid torsion $-33.4 \pm 9.9^{\circ}(-51.5-8.2)$, and distal torsion $8.3 \pm 3.2^{\circ}(1.2-$ 14.7) for distal torsion. Mean torsion measured in male and female subjects separately is given in Table 1. Mean total torsion was significantly greater in women than in men. Regarding segmental torsion, the greatest difference between men and women was found for proximal torsion. Gender differences were assumed and therefore not related to a hypothesis.

According to the Landis and Koch grading system [14], we found almost perfect intra-rater agreement for total torsion for all investigators and in three of four investigators for proximal torsion and two of four for mid torsion. The other ICCs were graded as substantial agreement. Inter-rater agreement (ICC) for average measures was found to be almost perfect for all measurements and almost perfect for total femoral torsion, proximal torsion, and mid torsion when evaluating the single measures. None of the ICC had to be graded as light, fair or moderate (detailed and inter-rater ICC values are provided in Table 2).

\section{Discussion}

This study confirmed our hypothesis that the segmental measurement protocol evaluating femoral torsion in CT is a reliable tool that allows segmental torsion analysis according to the anatomic segments of long bones. In addition to measuring total torsion, segmental femoral torsion was determined for the proximal metaphysis, the diaphysis, and the distal metaphysis separately. In agreement with the literature we found almost perfect intra- and inter-rater agreement for total femoral torsion measurements [15]. By calculating the ICC we were able to show that the measurement protocol introduced is a reliable tool for evaluation of segmental torsion, as we found substantial to almost perfect intra- and interobserver agreement for all parameters.

Radiological determination of femoral torsion by means of two plain x-rays was already described by Rippstein in 1955 [16]. Later, Weiner et al. described a method for measuring total femoral torsion in CT [17]. Currently, in clinical practice total femoral torsion is determined in the transverse plane between the femoral neck (proximal femur) and the posterior condylar line (distal femur) [18]. Later, Kim et al. published a method differentiating supratrochanteric and infratrochanteric torsion in CT [19], measured between the cervical axis, an intertrochanteric axis and the condylar axis. They concluded that de-rotational osteotomies should be performed at the site of the deformity to prevent the development of another lever arm disease after correction. As a further advancement, the aim of this study was to evaluate a tool that can be used to assess the torsion of three different femoral segments in CT. The concept of segmental analysis of femoral torsion was first introduced by Seitlinger et al. [10] and its reliability was further assessed for use in CT scans in this study. Similar to our data, Seitlinger et al. already found nearly perfect inter-rater agreement. However, their data were based only on measurements in MRI [10]. The reliability of total torsion measurement in CT was evaluated in previous studies, and it was reported that depending on the method used significant differences in torsion measurements and their reliability can be found $[15,18]$. Recently, Kaiser et al. reported that total femoral torsion measurements can differ by more than $10^{\circ}$, depending on the measurement technique used [15]. In this study we used the technique described by Murphy et al. [11], as high intra- (ICC: 0.95-0.98) and inter-observer agreement (ICC: 0.93 ) has been reported for this method [15]. In our collective we found mean total femoral torsion to be $22.6 \pm 8.7^{\circ}$, which lies between the measurements published by Kaiser et al. $\left(17.5 \pm 7.0^{\circ}\right)[15]$ and Sugano et al. $\left(26.0 \pm 9.1^{\circ}\right)$ [18]. Compared to the controls in the study by Seitlinger et al., we found higher mean torsion values (total femoral torsion: $22.6^{\circ}$ vs. $10.7^{\circ}$; proximal torsion: $47.7^{\circ}$ vs. $29.9^{\circ}$; mid torsion:
Table 1 Degree of femoral torsion in the 15 male and 15 female cases. Data are given as: mean \pm SD (range). Differences between the two groups were assessed with the t-test

\begin{tabular}{lccc}
\hline Torsion & Male & Female & $p$-value \\
\hline Total & $18.3^{\circ} \pm 7.3(10.2-34.3)$ & $26.8^{\circ} \pm 8.0(12.7-42.8)$ & 0.005 \\
Proximal & $44.7^{\circ} \pm 10.1(27.6-64.5)$ & $50.8^{\circ} \pm 10.5(32.0-62.4)$ & 0.113 \\
Mid & $-34.9^{\circ} \pm 9.5(-51.5--18.8)$ & $-31.8^{\circ} \pm 10.4(-44.2--8.2)$ & 0.397 \\
Distal & $8.6^{\circ} \pm 3.5(2.3-14.7)$ & $8.0^{\circ} \pm 3.0(1.2-12.0)$ & 0.665 \\
\hline
\end{tabular}


Table 2 Intra- and inter-rater agreement for total femoral torsion and for segmental torsion. Data given as: ICC (95\% confidence interval). ICC were graded according to Landis and Koch [14]: $\left({ }^{++}\right)$"almost perfect" $\left({ }^{+}\right)$"substantial"

\begin{tabular}{|c|c|c|c|c|c|c|}
\hline \multirow{2}{*}{$\begin{array}{l}\text { Torsion } \\
\text { Measurement }\end{array}$} & \multicolumn{4}{|c|}{ Intra-rater agreement (ICC) } & \multicolumn{2}{|c|}{ Inter-rater agreement (ICC) } \\
\hline & Investigator 1 & Investigator 2 & Investigator 3 & Investigator 4 & $\begin{array}{l}\text { Single } \\
\text { measures }\end{array}$ & $\begin{array}{l}\text { Average } \\
\text { measures }\end{array}$ \\
\hline Total & $\begin{array}{l}0.957^{++} \\
(0.912-0.979)\end{array}$ & $\begin{array}{l}0.897^{++} \\
(0.791-0.950)\end{array}$ & $\begin{array}{l}0.919^{++} \\
(0,759-0.967)\end{array}$ & $\begin{array}{l}0.876^{++} \\
(0.759-0.939)\end{array}$ & $\begin{array}{l}0.967^{++} \\
(0.944-0.983)\end{array}$ & $\begin{array}{l}0.992++ \\
(0.985-0.996)\end{array}$ \\
\hline Proximal & $\begin{array}{l}0.742^{+} \\
(0.403-0.885)\end{array}$ & $\begin{array}{l}0.905^{++} \\
(0.811-0.953)\end{array}$ & $\begin{array}{l}0.872^{++} \\
(0.583-0.950)\end{array}$ & $\begin{array}{l}0.854^{++} \\
(0.716-0.928)\end{array}$ & $\begin{array}{l}0.904^{++} \\
(0.841-0.948)\end{array}$ & $\begin{array}{l}0.974^{++} \\
(0.955-0.987)\end{array}$ \\
\hline Mid & $\begin{array}{l}0.746^{+} \\
(0.442-0.883)\end{array}$ & $\begin{array}{l}0.937^{++} \\
(0.872-0.969)\end{array}$ & $\begin{array}{l}0.944^{++} \\
(0.888-0.973)\end{array}$ & $\begin{array}{l}0.789^{+} \\
(0.582-0.897)\end{array}$ & $\begin{array}{l}0.903^{++} \\
(0.840-0.948)\end{array}$ & $\begin{array}{l}0.974^{++} \\
(0.955-0.986)\end{array}$ \\
\hline Distal & $\begin{array}{l}0.684^{+} \\
(0.439-0.835)\end{array}$ & $\begin{array}{l}0.713^{+} \\
(0.483-0.852)\end{array}$ & $\begin{array}{l}0.762^{+} \\
(0.544-0.882)\end{array}$ & $\begin{array}{l}0.504^{+} \\
(0.190-0.726)\end{array}$ & $\begin{array}{l}0.643^{+} \\
(0.436-0.798)\end{array}$ & $\begin{array}{l}0.878^{++} \\
(0.756-0.940)\end{array}$ \\
\hline
\end{tabular}

$-33.4^{\circ}$ vs. $-27.4^{\circ}$; distal torsion: $8.3^{\circ}$ vs. $8.2^{\circ}$ ) [10]. One reason for this might be the orientation and thickness of the transverse slices, while another might be that although patients with fractures of the femur were excluded, it is possible that some patients had other underlying conditions. Besides proving the reliability of this measurement protocol, we also compared mean torsion values in men and women. Total torsion was seen to be significantly greater in female femora, which is in agreement with the literature [20]. The biggest gender difference in segmental torsion was found in the proximal femur.

With regard to the question of clinical relevance, the measurement method evaluated here gives a better understanding of torsional deformities and may improve treatment planning. When only total femoral torsion is measured, no differentiation can be made for a torsional deformity located at the proximal, diaphyseal or distal aspect of the femur. This, however, could be important information when planning a derotational osteotomy. Currently, debate still surrounds the ideal site for an osteotomy, which can be performed proximally or distally $[4,8,9,21,22]$. If an osteotomy is performed at the site of the deformity, normal anatomy can be restored [6]. Beyond the question of correct location of derotational osteotomies, it might also be speculated whether specific segmental torsional anomalies interact with hip or knee pathologies. For example, it was shown that increased femoral torsion is associated with a flatter, more dysplastic trochlea, which can lead to patellofemoral instability. This was particularly true for torsion located on the distal femur [3].

This was the first study to prove that the measurement technique introduced can be reliably used to analyze segmental torsion of femora in CT. The following limitations are acknowledged and might also serve as suggestions for future studies: This was a retrospective study with the weaknesses associated with such studies, and we were not able to analyze for the clinical impact of differences in segmental torsion such as gait parameters or joint disorders. Second, the number of patients included was too small to determine standard values for women and men that can be applied as a threshold between physiological variances of torsion and pathological torsion. Further studies will be needed to determine normal values for the healthy population and describe gender differences in more detail. Furthermore, due to the random selection of CT scan data from the university hospital's PACS, some patients may have had some kind of lower limb complaint. Nevertheless, a valid evaluation of reliability was possible. As only skeletally mature subjects were included, the changes in segmental torsion during growth need to be further analyzed.

\section{Conclusion}

Evaluation of the segmental torsion of the femur allows indepth analysis of femoral alignment. High reliability was shown for this measuring method in computed tomography, which can be deployed when studying interdependencies between joint pathologies and torsional deformities or when planning the site for an osteotomy.

Acknowledgements Open access funding provided by University of Innsbruck and Medical University of Innsbruck.

\section{Compliance with ethical standards}

Conflict of interest The authors declare that they have no competing interests.

Open Access This article is distributed under the terms of the Creative Commons Attribution 4.0 International License (http:// creativecommons.org/licenses/by/4.0/), which permits unrestricted use, distribution, and reproduction in any medium, provided you give appropriate credit to the original author(s) and the source, provide a link to the Creative Commons license, and indicate if changes were made. 


\section{References}

1. Fabricant PD, Fields KG, Taylor SA, Magennis E, Bedi A, Kelly BT (2015) The effect of femoral and acetabular version on clinical outcomes after arthroscopic femoroacetabular impingement surgery. J Bone Joint Surg Am 97(7):537-543. doi: 10.2106/JBJS.N.00266

2. Carty CP, Walsh HP, Gillett JG, Phillips T, Edwards JM, deLacy M, Boyd RN (2014) The effect of femoral derotation osteotomy on transverse plane hip and pelvic kinematics in children with cerebral palsy: a systematic review and meta-analysis. Gait Posture 40(3): 333-340. doi:10.1016/j.gaitpost.2014.05.066

3. Liebensteiner MC, Ressler J, Seitlinger G, Djurdjevic T, El Attal R, Ferlic PW (2016) High femoral anteversion is related to femoral Trochlea dysplasia. Arthroscopy 32(11):2295-2299. doi:10.1016/j. arthro.2016.03.023

4. Delgado ED, Schoenecker PL, Rich MM, Capelli AM (1996) Treatment of severe torsional malalignment syndrome. J Pediatr Orthop 16(4):484-488

5. Hinterwimmer S, Minzlaff P, Saier T, Niemeyer P, Imhoff AB, Feucht MJ (2014) Biplanar supracondylar femoral derotation osteotomy for patellofemoral malalignment: the anterior closedwedge technique. Knee Surg Sports Traumatol Arthrosc 22(10): 2518-2521. doi:10.1007/s00167-014-2993-6

6. Paley D, Herzenberg JE, Tetsworth K, McKie J, Bhave A (1994) Deformity planning for frontal and sagittal plane corrective osteotomies. Orthop Clin North Am 25(3):425-465

7. Paley D, Tetsworth K (1992) Mechanical axis deviation of the lower limbs. Preoperative planning of uniapical angular deformities of the tibia or femur. Clin Orthop Relat Res 280:48-64

8. Fouilleron N, Marchetti E, Autissier G, Gougeon F, Migaud H, Girard J (2010) Proximal tibial derotation osteotomy for torsional tibial deformities generating patello-femoral disorders. Orthop Traumatol Surg Res 96(7):785-792. doi:10.1016/j.otsr. 2010.04.008

9. Pirpiris M, Trivett A, Baker R, Rodda J, Nattrass GR, Graham HK (2003) Femoral derotation osteotomy in spastic diplegia. Proximal or distal? J Bone Joint Surg (Br) 85(2):265-272

10. Seitlinger G, Moroder P, Scheurecker G, Hofmann S, Grelsamer RP (2016) The contribution of different femur segments to overall femoral torsion. Am J Sports Med 44(7):1796-1800. doi:10.1177/ 0363546516639945

11. Murphy SB, Simon SR, Kijewski PK, Wilkinson RH, Griscom NT (1987) Femoral anteversion. J Bone Joint Surg Am 69(8): $1169-1176$

12. Hallgren KA (2012) Computing inter-rater reliability for observational data: an overview and tutorial. Tutor Quant Methods Psychol 8(1):23-34. doi:10.1016/j.biotechadv.2011.08.021.Secreted

13. Koo TK, Li MY (2016) A guideline of selecting and reporting Intraclass correlation coefficients for reliability research. J Chiropr Med 15(2):155-163. doi:10.1016/j.jcm.2016.02.012

14. Landis JR, Koch GG (1977) The measurement of observer agreement for categorical data. Biometrics 33(1):159-174. doi:10.2307/ 2529310

15. Kaiser P, Attal R, Kammerer M, Thauerer M, Hamberger L, Mayr R, Schmoelz W (2016) Significant differences in femoral torsion values depending on the CT measurement technique. Arch Orthop Trauma Surg 136(9):1259-1264. doi:10.1007/s00402-016-2536-3

16. Rippstein $\mathrm{J}$ (1955) Determination of the antetorsion of the femur neck by means of two x-ray pictures. Z Orthop Ihre Grenzgeb 86(3):345-360

17. Weiner DS, Cook AJ, Hoyt WA Jr, Oravec CE (1978) Computed tomography in the measurement of femoral anteversion. Orthopedics 1(4):299-306

18. Sugano N, Noble PC, Kamaric E (1998) A comparison of alternative methods of measuring femoral anteversion. J Comput Assist Tomogr 22(4):610-614

19. Kim HY, Lee SK, Lee NK, Choy WS (2012) An anatomical measurement of medial femoral torsion. J Pediatr Orthop B 21(6):552557. doi:10.1097/BPB.0b013e328355e5f1

20. Nguyen AD, Shultz SJ (2007) Sex differences in clinical measures of lower extremity alignment. J Orthop Sports Phys Ther 37(7): 389-398. doi:10.2519/jospt.2007.2487

21. Bruce WD, Stevens PM (2004) Surgical correction of miserable malalignment syndrome. J Pediatr Orthop 24(4):392-396

22. Dickschas J, Harrer J, Reuter B, Schwitulla J, Strecker W (2015) Torsional osteotomies of the femur. J Orthop Res 33(3):318-324. doi:10.1002/jor.22758 